\title{
Encapsidation of Potyviral RNA in Various Forms of Transgene Coat Protein Is Not Correlated with Resistance in Transgenic Plants
}

\author{
John Hammond and Margaret M. Dienelt \\ United States Department of Agriculture, Agricultural Research Service, United States National Arboretum, \\ Floral and Nursery Plants Research Unit, Beltsville, MD 20705, U.S.A. \\ Accepted 11 August 1997.
}

\begin{abstract}
Transgenic plants expressing either bean yellow mosaic potyvirus or chimeric potyvirus coat protein $(\mathrm{CP})$ were inoculated with various potyviruses. Antigen-coated plate, indirect enzyme-linked immunosorbent assay and immunoelectron microscopy of virus purified from transgenic plants showed that progeny virions contained from $<1 \%$ to as much as $\mathbf{2 5 \%}$ transgenic CP. Different levels of transcapsidation may reflect the extent of compatibility between transgene $\mathrm{CP}$ and the viral $\mathrm{CP}$.
\end{abstract}

Additional keywords: aphid transmissibility, phenotypic mixing.

Transcapsidation, or phenotypic mixing, describes the coating of the RNA of one virus or isolate either partially or completely with the coat protein $(\mathrm{CP})$ of another virus or isolate (Rochow 1970). Because the CP of potyviruses and other virus groups determines vector specificity, transcapsidation with transgene $\mathrm{CP}(\mathrm{tCP})$ could result in introduction of the virus to a new ecological niche (Hull 1990; de Zoeten 1991). Farinelli et al. (1992), Lecoq et al. (1993), and Maiss et al. (1994) have demonstrated partial transcapsidation of potyviruses with heterologous $\mathrm{CP}$ in transgenic plants. Lecoq et al. (1993) further demonstrated aphid transmissibility of a nonaphid-transmitted (NAT) isolate of zucchini yellow mosaic virus (ZYMV) as a result of incorporation of tCP from an aphid-transmissible isolate of plum pox virus (PPV).

Re-encapsidation of incoming genomic RNA in tCP has been suggested as one possible mechanism of CP-mediated resistance (Beachy et al. 1990). Compatibility between tCP and viral RNA and/or viral CP is presumed to be necessary for either transcapsidation or re-encapsidation. We examined

Corresponding author: John Hammond

E-mail: jhammond@asrr.arsusda.gov

Mention of a trademark, warranty, proprietary product, or vendor does not constitute a guarantee by the U.S. Department of Agriculture and does not imply its approval to the exclusion of other products or vendors that may also be suitable.

This article is in the public domain and not copyrightable. It may be freely reprinted with customary crediting of the source. The American Phytopathological Society, 1997. the relationship between transcapsidation and the degree of virus resistance in transgenic plants expressing $\mathrm{CP}$ of either bean yellow mosaic virus (BYMV) or chimeric CPs. The chimeric CPs contain the N-terminal domain of BYMV and C-terminal domains of either potato virus $\mathrm{Y}$ (PVY) or ZYMV. The transgenic lines tested show varying degrees of resistance to initial infection and/or recovery from infection (Hammond and Kamo 1993, 1995a; Hammond 1993; Hammond et al. 1995).

The BYMV transgene constructs CP-1 and CP-2 and chimeric constructs BYMV/PVY and BYMV/ZYMV (referred to as Pe and ZY, respectively; Fig. 1) and transformation of Nicotiana benthamiana were described previously (Hammond and Kamo 1995a). The chimeric construct Pe was derived from a PVY isolate originally described as pepper mottle virus (PepMoV; Dougherty et al. 1985). Each tCP contains the Nterminal epitope from BYMV-GDD that is recognized by strain-specific monoclonal antibody (MAb) PTY 43, which reacts with BYMV-GDD but not other isolates of BYMV or other potyviruses (Jordan and Hammond 1991; Jordan 1992). The tCPs were thus distinguishable from all potyvirus CPs except BYMV-GDD. Cross-reactive MAb PTY 1 reacts with all of the potyviruses used in these experiments (Jordan and Hammond 1991).

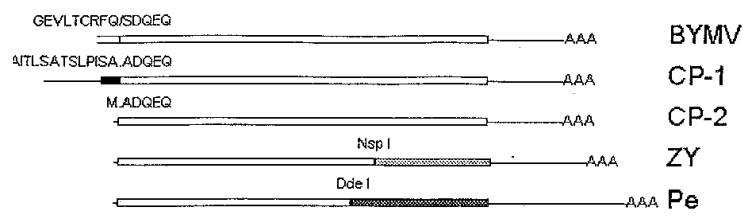

Fig. 1. Comparison of the transgene constructs to the bean yellow mosaic virus (BYMV) coat protein (CP) gene. The amino acid sequence of $\mathrm{BYMV-GDD}$ at the NIb/CP junction is shown above the open bar representing the carboxyterminal portion of the BYMV-GDD polyprotein. $\mathrm{CP}-1$ has the noncoding leader sequence from nopaline synthase; the CP-1 amino acid sequence shows the initial Met and 13 additional residues also derived from nopaline synthase, plus a Ser to Ala change at the first residue from the BYMV-CP. CP-2 and the chimeric ZY and Pe constructs each share a short synthetic leader and initial Met, as well as the Ser to Ala change present in CP-1. The NspI and DdeI sites used to create the $\mathrm{ZY}$ and Pe constructs are also indicated, with the zucchini yellow mosaic virus and potato virus Y CP domains shaded. Untranslated and coding sequences are shown approximately to scale. 
Plants expressing tCPs were challenge inoculated with BYMV-Scott (Hobbs and McLaughlin 1990) or six other potyviruses; i.e., BYMV-Ideal A, clover yellow vein virus Pratt isolate (CYVV-P), PepMoV NC 165, PVY-3, tobacco etch virus (TEV-NAT), and turnip mosaic virus (TuMV) PV 177, which were propagated as described (Hammond and Lawson 1988). Sap extracts of infected plants (approximately $1 \mathrm{~g}$ per $50 \mathrm{ml}$ of $1 \% \mathrm{~K}_{2} \mathrm{HPO}_{4}$ ) were used to inoculate transgenic plant lines CP-1 30, CP-1 33, CP-1 79, CP-2 37, CP-2 44, Pe 9, Pe $46, \mathrm{ZY} 34$, and ZY 43. Virus resistance of transgenic lines was determined as described (Hammond and Kamo 1993, 1995a, $1995 \mathrm{~b}$ ), and virions were purified from systemically infected tissue of both transgenic and nontransgenic (NT) plants (Hammond and Lawson 1988).

Transcapsidation of progeny virions purified from transgenic plants was detected in all cases by probing Western blots (immunoblots) with MAb PTY 43. No reaction was observed with virus from NT plants except for purified BYMVGDD. Reactions with virus from transgenic plants varied from strong (BYMV-Ideal A from CP-1 30) to weak (PepMoV from Pe 9). MAb PTY 1 reacted with all preparations tested from both tCP and NT plants (data not shown).

The proportion of tCP incorporated into virus preparations was estimated by antigen-coated plate, indirect enzyme-linked immunosorbent assay (ACP-ELISA) (Jordan and Hammond 1991). We assumed that the reaction of PTY 43 with its epi-

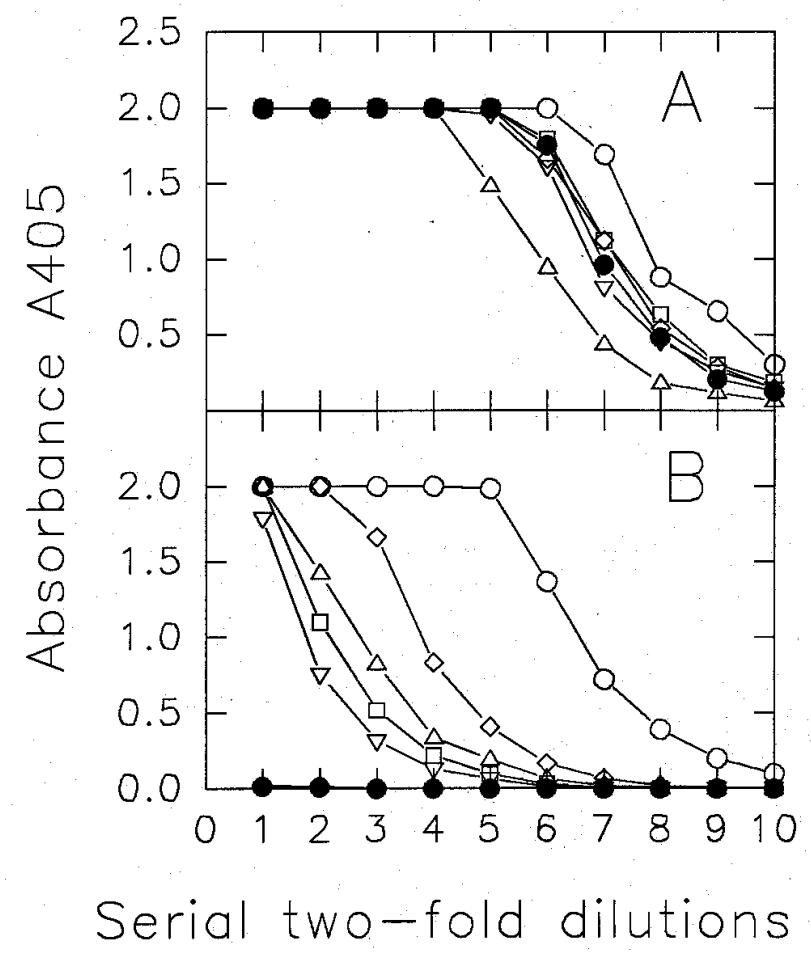

Fig. 2. Antigen-coated plate, indirect enzyme-linked immunosorbent assay (ACP-ELISA) analysis (absorbance at $405 \mathrm{~nm}$ ) of purified virus preparations. Key: Bean yellow mosaic virus (BYMV)-GDD from $(O)$ nontransgenic (NT) plants; BYMV-Ideal A from $(\nabla)$ ZY 34, ( $\square)$ Pe $9,(\triangle)$ CP-2 37, ( $\diamond)$ CP-2 44, and (৩) NT plants. ELISA was performed as described (Jordan and Hammond 1991) with serial twofold dilutions of virus starting at $2 \mu \mathrm{g} / \mathrm{ml}$ (dilution 1) with monoclonal antibodies PTY 1 (A) and PTY 43 (B). There is an absence of reaction of PTY 43 with BYMV-Ideal A purified from NT plants. tope in chimeric tCP is quantitatively equivalent to that with BYMV-GDD. Serial twofold dilutions of each virus preparation $(2 \mu \mathrm{g} / \mathrm{ml})$ were reacted with PTY 1 and PTY 43 (Fig. 2). The ratios of the ELISA reactions of the two MAbs at the midpoint of the curve were used to estimate the degree of transcapsidation, with BYMV-GDD as the $100 \%$ control.

Although ACP-ELISA is only semi-quantitative (Hammond and Lawson 1988), it is the most appropriate form of ELISA for specific detection of the tCPs (Jordan and Hammond 1991). ELISA (Fig. 2) and Western blot (data not shown) results were comparable. As summarized in Table 1, the chimeric tCPs were incorporated into virions less efficiently than the BYMV tCPs. The greatest degree of transcapsidation was observed from BYMV-infected plants expressing $\mathrm{CP}-1$ or $\mathrm{CP}-2$, and the least in PepMoV-infected plants of line Pe 9 (Table 1).

In some of the transgenic lines there was resistance against potyvirus infection. The most extreme resistance to BYMV was in line CP-1 30. In this line many plants escaped infection, and most BYMV-infected plants recovered completely

Table 1. Incorporation of transgene coat protein (tCP) into virions compared to protection from virus infection

\begin{tabular}{|c|c|c|}
\hline $\begin{array}{l}\text { Virus isolate/ } \\
\text { plant line }^{\mathrm{a}}\end{array}$ & $\begin{array}{l}\text { Transgene } \\
\text { incorporation }^{b}\end{array}$ & Degree of protection ${ }^{c}$ \\
\hline \multicolumn{3}{|l|}{ BYMV-Ideal A } \\
\hline CP-1 30 & ca. $25 \%$ & Initial $^{\mathrm{d}}+$ recovery $^{\mathrm{e}}$ \\
\hline CP-1 33 & 12.5 to $25 \%$ & Recovery \\
\hline CP-2 37 & 12.5 to $25 \%$ & Partial recovery ${ }^{\mathrm{f}}$ \\
\hline CP-2 44 & 12.5 to $25 \%$ & Recovery \\
\hline ZY 34 & 3 to $6 \%$ & Recovery \\
\hline Pe 9 & 6 to $12.5 \%$ & $\begin{array}{l}\text { Initial protection (dilute inocula } \\
\text { only) }\end{array}$ \\
\hline \multicolumn{3}{|l|}{ BYMV-Scott } \\
\hline CP-1 79 & 12.5 to $25 \%$ & Recovery \\
\hline \multicolumn{3}{|l|}{ CYVV-P } \\
\hline CP-2 44 & 3 to $6 \%$ & $\mathrm{ND}^{\mathrm{h}}$ \\
\hline \multicolumn{3}{|l|}{ TuMV PV 177} \\
\hline CP-1 30 & 6 to $12.5 \%$ & Slight delay ${ }^{\mathrm{i}}$ \\
\hline ZY 43 & 1.5 to $3 \%$ & Slight delay \\
\hline \multicolumn{3}{|l|}{ TEV-NAT } \\
\hline CP-1 30 & 6 to $12.5 \%$ & None \\
\hline \multicolumn{3}{|l|}{ PVY-3 } \\
\hline CP-1 30 & 6 to $12.5 \%$ & None \\
\hline ZY 34 & 0.75 to $1.5 \%$ & None \\
\hline Pe 46 & 0.75 to $1.5 \%$ & Partial recovery \\
\hline \multicolumn{3}{|c|}{ PepMoV NC 165} \\
\hline Pe 9 & $<0.75 \%$ & None \\
\hline
\end{tabular}

a See text for definitions of all abbreviations.

${ }^{b}$ tCP incorporation as a proportion of the virion $\mathrm{CP}$ was estimated from antigen-coated plate, indirect enzyme-linked immunosorbent assay, as described in the text and shown in Figure 2.

c Summary of data from both published (Hammond and Kamo 1993, 1995a) and unpublished results.

${ }^{\mathrm{d}}$ Some plants escaped infection after inoculation under conditions in which all of the control nontransgenic plants were infected.

e Most or all initially infected plants subsequently developed leaves with reduced symptoms and then symptomless leaves in which virus could not be detected.

${ }^{\mathrm{f}}$ Plants showing partial recovery produced leaves with reduced symptoms and lower virus titers compared with controls, but did not proceed to full recovery.

${ }^{\mathrm{g}}$ Line Pe 9 showed initial resistance to low concentrations (i.e., $2 \mu \mathrm{g} / \mathrm{ml}$ ) of BYMV-Ideal A, but not to more concentrated inocula $(20 \mu \mathrm{g} / \mathrm{ml})$.

${ }^{\mathrm{h}}$ Degree of resistance to CYVV-P has not yet been determined.

i A 2 to 3 day delay in symptom expression was observed in these lines, but there was no apparent reduction in symptom severity or ultimate virus titer. 
(Table 1; Hammond and Kamo 1995a). Other lines exhibited intermediate levels of resistance, manifest as either delay of symptoms or recovery. There were also lines that were fully susceptible to one or more of the potyviruses tested.

However, there was no correlation between tCP incorporation and the extent of resistance. For example, the tCP was approximately $25 \%$ of the virus CP of BYMV-Ideal A purified from line CP-1 30; in this line there was both resistance to initial BYMV infection and recovery of infected plants. The tCP of CP-1 30 was also incorporated to a high level in particles of TEV, PVY, and TuMV, against which there was little or no resistance (Table 1; Hammond and Kamo 1995a). The PVY-infected plants of lines CP-1 30 and Pe 46 also illustrate the absence of correlation between transcapsidation and resistance. The tCP of CP-1 30 was incorporated to 6 to $12.5 \%$ of virion $\mathrm{CP}$, although the plants were fully susceptible to PVY; in contrast, the Pe tCP was only 0.75 to $1.5 \%$ of PVY virion $\mathrm{CP}$ from line Pe 46 despite there being partial recovery from PVY in this line (Table 1). We assume that transcapsidation and re-encapsidation are similar, and therefore the lack of correlation between transcapsidation and resistance suggests that tCP encapsidation of viral RNA entering a new cell does not contribute significantly to systemic resistance to potyviruses.

To establish that the tCP detected in the purified virus preparations was due solely to transcapsidation, and not to contamination of virus cultures or recombination between the infecting potyvirus and the transgene, the purified preparations were used to inoculate NT plants. Infection of NT $N$. benthamiana, $N$. glutinosa, and N. tabacum with each of the partially transcapsidated virus preparations yielded only symptoms typical of the wild-type viruses, and no ELISA reaction with MAb PTY 43 was obtained from plants inoculated with transcapsidated virus. In contrast, BYMV-GDD was readily detected in NT $N$. benthamiana inoculated with a 1:49 mixture of sap extracts of BYMV-GDD- and PVY-infected plants, showing that (i) BYMV-GDD was able to establish an infection in the presence of a considerable excess of another virus and (ii) that Western blot and ELISA results were not due to contamination with BYMV-GDD or recombination between the transgene and the infecting virus (BYMV does not infect either N. glutinosa or N. tabacum).

The detection of transcapsidated virions by ELISA could either indicate that a proportion of the virions were completely transcapsidated, or that most or all particles were partially encapsidated in tCP. To address this issue we used immunoelectron microscopy. This technique revealed that virions of BYMV-GDD were uniformly decorated with gold particles after reaction with MAb PTY 43 and gold-labeled goat antimouse antibodies (Fig. 3A). The specificity of the assay was confirmed by the absence of gold particles on other potyvirus virions isolated from NT plants (Fig. 3B,C). Transcapsidated virions purified from transgenic plants infected with BYMVIdeal A, PVY, PepMoV, TEV, and TuMV (Fig. 3D-K, and not shown) exhibited gold particle decoration that varied between virions of each preparation (e.g., Fig. $3 \mathrm{H}, \mathrm{K}$ ). The transcapsidated particles showing the least labeling were those of Pep$\mathrm{MoV}$ isolated from line Pe 9; most particles were not labeled at all, and others very sparsely labeled, in agreement with the ELISA estimate of $<0.75 \%$ tCP incorporation (Table 1). At the other extreme, virions of BYMV-Ideal A isolated from line
CP-1 30 showed extensive labeling. However, in none of the preparations was there a subpopulation of completely labeled virions. It is therefore likely that transcapsidation involves intercalation of tCP among the virus-encoded CP subunits. Localized antibody and gold particle interaction along the length of virions (e.g., Fig. 3F,K) suggests that there is preferential association of homologous subunits (either tCP or virusencoded $\mathrm{CP}$ ) rather than random assembly.

Besides illustrating the distribution of transcapsidated virions, the immunoelectron microscopy also produced information about the stability of these hybrid virus particles. The gold particles that were not associated with virions (Fig. $3 \mathrm{~A}, \mathrm{D}, \mathrm{F}, \mathrm{I})$ presumably represent the interaction of MAb PTY 43 with subunits from dissociated or degraded virions.

Our ability to distinguish tCP from viral-derived $\mathrm{CP}$ allowed us to detect and quantify transcapsidation in all potyvirus:tCP combinations examined. Chimeric tCPs were incorporated to a lesser extent than BYMV tCP, but were apparently correctly folded and assembled. Virions containing tCP banded with wild-type virions on $\mathrm{CsCl}$ gradients (data not shown), but the apparent extent of free subunits following storage of preparations including ZY tCP suggests that particle stability was affected. As single amino acid changes in Johnsongrass mosaic potyvirus and tobacco mosaic virus CPs significantly affect subunit:subunit interactions and particle stability (Jagadish et al. 1993; Culver et al. 1994), chimeric tCPs might have a similar particle destabilizing effect. Differences in the extent of incorporation of different tCPs may reflect a greater compatibility between the viral CPs and the BYMV tCPs than with the chimeric tCPs.

Potential disadvantages of CP-mediated resistance (reviewed by Beachy et al. 1990) have been described. The ability of transcapsidation in potyvirus CP-transgenic plants (Farinelli et al. 1992; Lecoq et al. 1993; Maiss et al. 1994; this report) to alter viral host range, pathogenicity, or epidemiology (Hull 1990; de Zoeten 1991) must be compared with the effects of transcapsidation in mixed infections. While Lecoq et al. (1993) have demonstrated heterologous encapsidation and consequent viral transmission from a combination (PPV tCP and ZYMV) unlikely to occur naturally, the ZYMV-NAT cultures derived as a result were not further aphid transmissible. This confirms the prediction that any such transmission would be restricted to the initial passage from the transgenic crop (Hull 1990). Naturally occurring transcapsidation has been reported in mixed infections of several virus groups (Creamer and Falk 1990; Rochow 1970; Sarkar 1969; Bourdin and Lecoq 1991; Nelson and Wheeler 1979). Considering the widespread occurrence of natural mixed infections (especially in vegetatively propagated crops; Alper et al. 1984; Brunt and Phillips 1980), the broad virus transmission capabilities of many common polyphagous aphid vector species (Kennedy et al. 1962), and the most likely scenario of deployment of tCPs of viruses that naturally infect a transgenic crop, the added risks from deployment of potyvirus CP-transgenic plants seem minimal. Prevention or minimization of transcapsidation would reduce such risks even further.

Our results suggest that significant resistance can be obtained with chimeric tCPs that are incorporated into virions to a lesser extent than wild-type CPs and that may even destabilize virions. Virion destabilization would likely result in reduced virus transmission. We are examining other alterations 


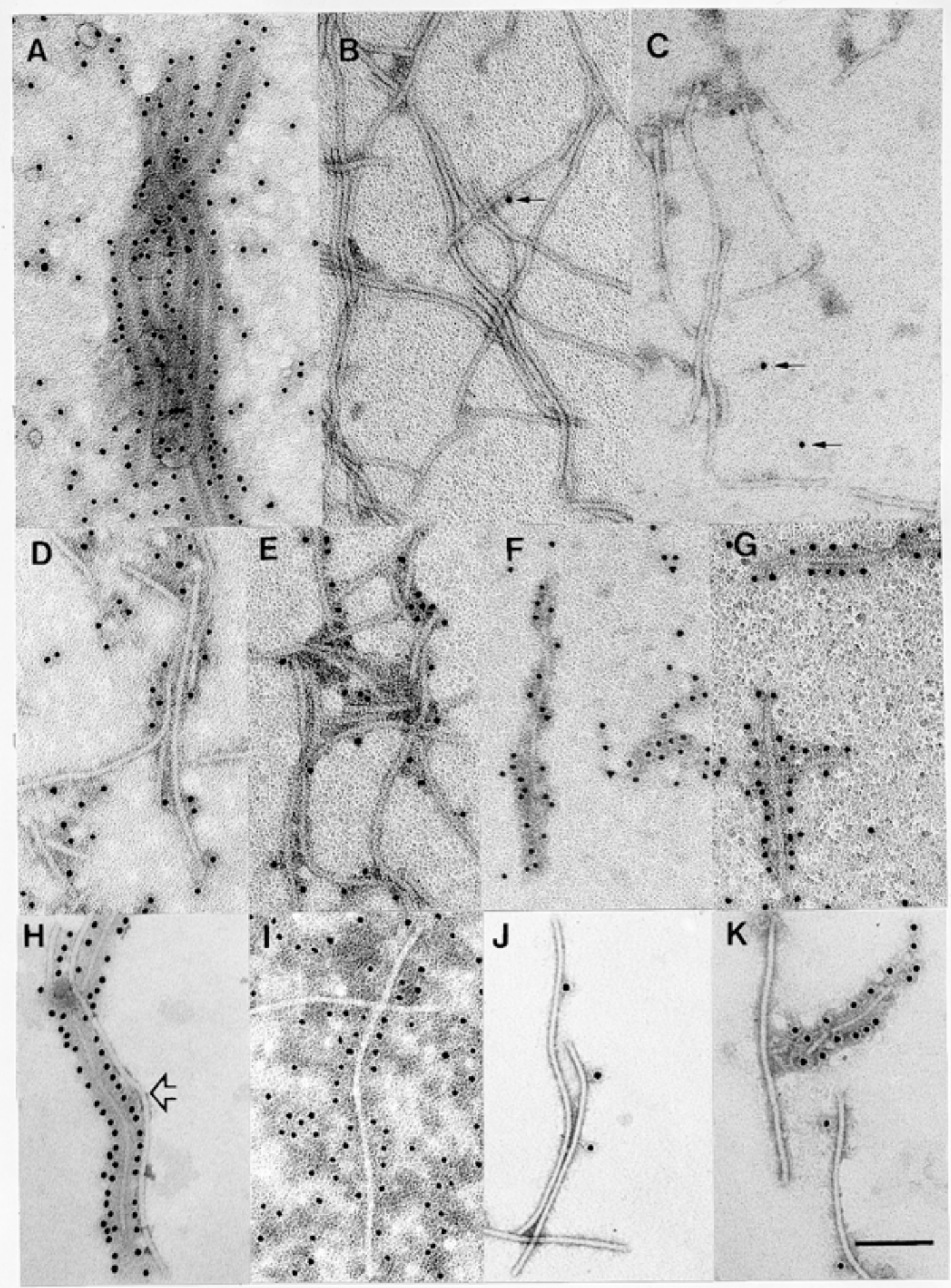

Fig. 3. Immunoelectron microscopy of virus particles purified from Nicotiana benthamiana, after reaction with a 1:500 dilution of monoclonal antibody PTY 43 and $10 \mathrm{~nm}$ gold-labeled goat anti-mouse conjugate. A, Bean yellow mosaic virus (BYMV)-GDD from nontransgenic (NT) plants; B, BYMVIdeal A from NT plants; C, potato virus Y (PVY) from NT plants; D, BYMV-Ideal A from Pe 9; E, BYMV-Ideal A from CP-1 30; F, BYMV-Ideal A from CP-1 33; G, BYMV-Scott from CP-1 79; H, pepper mosaic virus (PepMoV) from Pe 9; I, PVY from ZY 34; J, PVY from Pe 46; and K, tobacco etch virus (TEV) from CP-1 30. Complete labeling of BYMV-GDD and presence of some free gold particles in A compares with the absence of labeling and little free gold (arrows) in $\mathbf{B}$ and $\mathbf{C}$, partial labeling of virions and some free gold in $\mathbf{D}-\mathbf{K}$, extensive labeling of three virions or fragments and absence of label from another virion (open arrow) in $\mathbf{H}$, and the extensive labeling of one virion fragment, two gold particles on a second, and none on a third in $\mathbf{K}$. Bar $=150 \mathrm{~nm}$. 
to potyvirus tCP to determine whether further minimization of transcapsidation (or maximization of virion destabilization) is compatible with significant resistance.

\section{ACKNOWLEDGMENTS}

We wish to thank Katerina Serlemitsos, Karen Green, and Chris Lee for excellent technical assistance, and G. A. de Zoeten, R. A. Owens, M. Laimer da Câmara Machado, and H. Steinkellner for critical reading of the manuscript.

\section{LITERATURE CITED}

Alper, M., Salomon, R., and Loebenstein, G. 1984. Gel electrophoresis of virus-associated polypeptides for detecting viruses in bulbous irises. Phytopathology 74:960-962.

Beachy, R. N., Loesch-Fries, S., and Tumer, N. E. 1990. Coat proteinmediated resistance against virus infection. Annu. Rev. Phytopathol. 28:451-474.

Bourdin, D., and Lecoq, H. 1991. Evidence that heteroencapsidation between two potyviruses is involved in aphid transmission of a nonaphid-transmissible isolate from mixed infections. Phytopathology 81: $1459-1464$.

Brunt, A. A., and Phillips, S. 1980. The detection, separation from naturally occurring complexes, and partial characterization of four aphidborne viruses infecting bulbous iris. Acta Hortic. 109:503-508.

Creamer, R., and Falk, B. W. 1990. Direct detection of transcapsidated barley yellow dwarf luteoviruses in doubly infected plants. J. Gen. Virol. 71:211-217.

Culver, J. N., Stubbs, G., and Dawson, W. O. 1994. Structure-function relationship between tobacco mosaic virus coat protein and hypersensitivity in Nicotiana sylvestris. J. Mol. Biol. 242:130-138.

de Zoeten, G. A. 1991. Risk assessment: Do we let history repeat itself? Phytopathology 81:585-586.

Dougherty, W. G., Allison, R. F., Parks, T. D., Johnston, R. E., Feild, M. J., and Armstrong, F. B. 1985. Nucleotide sequence at the $3^{\prime}$ terminus of pepper mottle virus genomic RNA: Evidence for an alternative mode of potyvirus capsid protein gene organization. Virology 146: 282-291.

Farinelli, L., Malnoë, P., and Collet, G. 1992. Heterologous encapsidation of potato virus $\mathrm{Y}$ strain $\mathrm{O}\left(\mathrm{PVY}^{\mathrm{O}}\right)$ with the transgenic coat protein of PVY strain N $\left(\mathrm{PVY}^{\mathrm{N}}\right)$ in Solanum tuberosum cv. Bintje. Bio/ Technology 10:1020-1025.

Hammond, J. 1993. Transcapsidation in transgenic plants expressing bean yellow mosaic virus or chimeric coat proteins is not correlated with resistance to various potyviruses. (Abstr.) Phytopathology 83: 1349.

Hammond, J., and Kamo, K. K. 1993. Transgenic coat protein and antisense RNA resistance to bean yellow mosaic potyvirus. Acta Hortic. 336:171-178.

Hammond, J., and Kamo, K. K. 1995a. Resistance to bean yellow mo- saic virus (BYMV) and other potyviruses in transgenic plants expressing BYMV antisense RNA, coat protein, or chimeric coat proteins. Pages 369-389 in: Biotechnology and Plant Protection: Viral Pathogenesis and Disease Resistance. D. D. Bills and S. D. Kung, eds. World Scientific, Singapore.

Hammond, J., and Kamo, K. K. 1995b. Effective resistance to potyvirus infection conferred by expression of antisense RNA in transgenic plants. Mol. Plant-Microbe Interact. 8:674-682.

Hammond, J., and Lawson, R. H. 1988. An improved purification procedure for preparing potyviruses and cytoplasmic inclusions from the same tissue. J. Virol. Methods 20:203-217.

Hammond, J., Pühringer, H., Steinkellner, H., da Câmara Machado, A., and Laimer da Câmara Machado, M. 1995. Transcapsidation in transgenic plants expressing potyvirus coat proteins. (Abstr.) Phytopathology 85:1151.

Hobbs, H. A., and McLaughlin, M. R. 1990. A non-aphid-transmissible isolate of bean yellow mosaic virus-Scott that is transmissible from mixed infections with pea mosaic virus-204-1. Phytopathology 80: 268-272.

Hull, R. 1990. The use and misuse of viruses in cloning and expression in plants. Pages 443-457 in: Recognition and response in plant-virus interactions. R. S. S. Fraser, ed. NATO ASI Ser., Vol. H41. SpringerVerlag, Berlin.

Jagadish, M. N., Huang, D., and Ward, C. W. 1993. Site-directed mutagenesis of a potyvirus coat protein and its assembly in Escherichia coli. J. Gen. Virol. 74:893-896.

Jordan, R. L. 1992. Potyviruses, monoclonal antibodies, and antigenic sites. Arch. Virol. (suppl. 5):81-95.

Jordan, R. L., and Hammond, J. 1991. Comparison and differentiation of potyvirus isolates and identification of strain-, virus-, subgroup-specific and potyvirus group-common epitopes using monoclonal antibodies. J. Gen. Virol. 72:25-36.

Kennedy, J. S., Day, M. F., and Eastop, V. F. 1962. A Conspectus of Aphids As Vectors of Plant Viruses. Commonwealth Institute of Entomology, London.

Lecoq, H., Ravelonandro, M., Wipf-Scheibel, C., Monsion, M., Raccah, B., and Dunez, J. 1993. Aphid transmission of a non-aphid-transmissible strain of zucchini yellow mosaic potyvirus from transgenic plants expressing the capsid protein of plum pox potyvirus. Mol. Plant-Microbe Interact. 6:403-406.

Maiss, E., Koenig, R., and Lesemann, D.-E. 1994. Heterologous encapsidation of viruses in transgenic plants and in mixed infections. Pages 129-139 in: Proc. Int. Symp. Biosafety Results of Field Tests of Genetically Modified Plants and Microorganisms, 3rd. D. D. Jones, ed. University of California, Division of Agriculture and Natural Resources, Oakland.

Nelson, M. R., and Wheeler, R. E. 1979. Heterologous encapsidation of viral RNA in mixed infections of two potyviruses. (Abstr.) Phytopathology 69:918.

Rochow, W. F. 1970. Barley yellow dwarf virus: Phenotypic mixing and vector specificity. Science 167:875-878.

Sarkar, S. 1969. Evidence of phenotypic mixing between two strains of tobacco mosaic virus. Mol. Gen. Genet. 105:87-90. 